\title{
Primary Care Physicians' Knowledge of the Cardiovascular Effects of Diabetes Medications: Findings from an Online Survey
}

Jay H. Shubrook · Jonathan Pak · George Dailey

Received: February 19, 2020 / Published online: July 4, 2020

(C) The Author(s) 2020

\begin{abstract}
Introduction: Cardiovascular (CV) outcomes trial (CVOT) results have led to changes in indications for some glucose-lowering agents, with recommendations based on the presence of comorbidities.

Objective: This study aimed to understand internal medicine (IM) and family medicine (FM) physicians' knowledge of CVOTs and beliefs about type 2 diabetes mellitus (T2DM) medications, excluding insulin, for CV disease risk reduction.

Methods: WebMD, LLC, fielded a 23-item online survey from September 18 to 20, 2018, to 47,534 Medscape members (US IM and FM physicians) who were invited to participate via e-mail (quota $=500)$.
\end{abstract}

Digital Features To view digital features for this article go to https://doi.org/10.6084/m9.figshare.12445481.

J. H. Shubrook ( $₫)$

Touro University California, Vallejo, CA, USA

e-mail: jay.shubrook@tu.edu

J. Pak

Boehringer Ingelheim Pharmaceuticals, Inc.,

Ridgefield, CT, USA

G. Dailey

University of California, San Diego, CA, USA
Results: Of the 702 physicians who responded, 503 were eligible and completed the survey. Overall, $39 \%$ of respondents were not familiar with the 2018 American Diabetes Association treatment recommendations for those with T2DM and atherosclerotic CV disease. Respondents reported they were most familiar with TECOS (42\%), LEADER (39\%), EMPA-REG OUTCOME (33\%), and CANVAS (30\%). Many respondents did not know which CVOT showed superiority for major adverse CV events (26\%) or CV mortality (31\%). When provided with a list of seven treatment priorities, $33 \%$ of respondents ranked using T2DM medications with CV benefits as least important.

Conclusions: Findings from this 2018 survey suggest that there are knowledge gaps among IM and FM physicians regarding the results from CVOTs, with implications for the treatment of patients with T2DM and CV disease. 


\section{Graphical Abstract:}

The full article title:

Primary Care Physicians' Knowledge of the Cardiovascular Effects of

Diabetes Medications: Findings from an Online Survey

Jay H. Shubrook, Jonathan Pak, George Dailey

A survey of doctors' knowledge about how treatments for type 2 diabetes affect heart health

- The purpose of this plain language summary is to help you understand the findings from a recent survey

1 What did this survey look at?

- Type 2 diabetes is a condition where insulin doesn't work properly, leading to high blood sugar levels. - Many people with type 2 diabetes also have heart problems, or are at high risk of developing heart problems.

- Treatments are available to help lower blood sugar levels for people with type 2 diabetes.

- Researchers have done studies of these treatments to look at their effects on heart health.

- Researchers did an online survey of doctors. They wanted to find out how much doctors knew about 14 studies. These studies looked at how treatments for type 2 diabetes (other than insulin) affect heart health.

- This summary describes the results of the survey.

2 whot

took part in this survey?

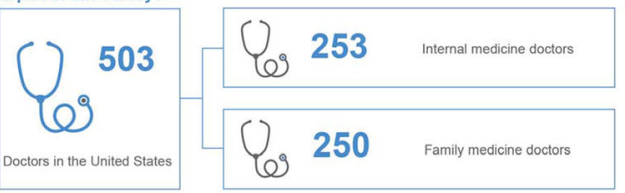

What were the results of this survey?

- The group of doctors had different levels of knowledge of the 14 studies

About 4 in 10 doctors were very familiar with studies best known by the group

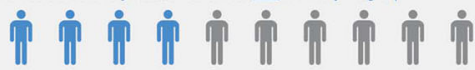

About 1 in 10 doctors were very familiar wth studies least known by the group

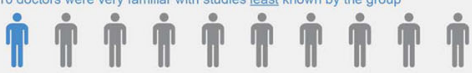

- All of the studies looked at a combined measure of heart health called Major Adverse Cardiovascular Events (MACE for short). MACE includes:

Dying from heart disease

Having a heart attack

Having a stroke
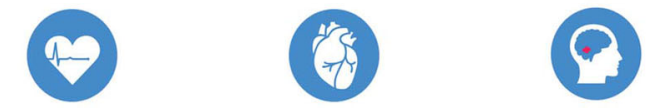

Some studies also looked at being admitted to the hospital for chest pain (known as angina: pronounced AN-juh-nuh)

- Fewer than half of the doctors knew which studies of type 2 diabetes treatments showed a lower risk of hert problems measured using MACE.

4 What was the main conclusion reported by the researchers?

- This survey suggests that some doctors in the United States need more information about the effects of type 2 diabetes treatments on heart health. type 2 diabetes to choose treatments.

5 Who supported this survey? - Boehringer Ingelheim \& Eli Lilly and Company Diabetes Alliance

Are there any plans for further surveys? - This survey is completed. who took part in this survey.

- There are no plans for further surveys.

Further information

You can find more information about type 2 diabetes and heart problems here: https://KnowDiabetesbyHeart.org/

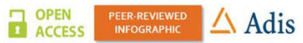

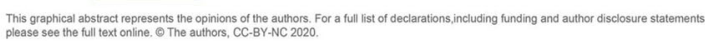

Summary prepared by Marissa Buttaro, MPH, Envision Pharma Group. Plain language services were funded by Boelringer Ingetheim. The

enignal authors of the manuscript reviewed this summary. 
Keywords: Cardiovascular receptor agonist; SGLT2 inhibitor; Type 2 diabetes mellitus

\section{Key Summary Points}

Cardiovascular outcomes trial (CVOT) results have led to changes in diabetes position statements and indications for some glucose-lowering agents

This study aimed to understand internal medicine (IM) and family medicine (FM) physicians' knowledge of CVOTs and beliefs about type 2 diabetes mellitus (T2DM) medications for CV disease risk reduction

Overall, 39\% of respondents were slightly/ not at all familiar with the 2018 American Diabetes Association treatment recommendations for those with T2DM and atherosclerotic CV disease

Most respondents were not familiar with the findings of recent CVOTs

Findings from this survey suggest that there are knowledge gaps among IM and FM physicians regarding the results from CVOTs

These gaps have implications for the treatment of patients with T2DM and CV disease

\section{INTRODUCTION}

Cardiovascular (CV) disease is the leading cause of mortality and morbidity for patients with type 2 diabetes mellitus (T2DM) [1, 2]. Internal medicine (IM) and family medicine (FM) physicians may appreciate the need to consider CV risk when treating patients with T2DM, but they may not be aware of the plethora of $\mathrm{CV}$ outcomes trials (CVOTs) that have been published over the last decade. In these CVOTs, the primary endpoint was most often a composite of time to first occurrence of CV death, nonfatal myocardial infarction, or non-fatal stroke, referred to as a 3-point major adverse CV event (3P-MACE) [3]; hospitalization for heart failure (HHF) was usually evaluated as a secondary outcome. To date, all completed CVOTs have confirmed the CV safety of the glucose-lowering agents tested and results from several CVOTs have shown that specific agents improve $\mathrm{CV}$ outcomes in patients with T2DM and CV disease $[4,5]$.

The first CVOT results (Table 1) published were for dipeptidyl peptidase 4 (DPP4) inhibitors (SAVOR-TIMI 53 [6], TECOS [7], and EXAMINE [8, 9]). These trials showed that compared with placebo, the addition of saxagliptin, sitagliptin, or alogliptin, respectively, to standard care diabetes therapy did not increase or decrease the event rate for the primary composite endpoint. More recently, CARMELINA [10] demonstrated the CV and renal safety of linagliptin compared with placebo in patients with T2DM and at high risk of $\mathrm{CV}$ and/or renal events. The first DPP4 inhibitor CVOT with an active comparator, CAROLINA, showed neither an increase nor a decrease in the risk of 3P-MACE for linagliptin compared with the sulfonylurea [11].

Among the sodium-glucose co-transporter 2 (SGLT2) inhibitors, EMPA-REG OUTCOME was the first CVOT to demonstrate a reduced risk of 3P-MACE as well as reduced risk of CV mortality for empagliflozin vs. placebo added to standard care $[12,13]$. The trial also showed that empagliflozin reduced the risk of HHF. This was followed by the CANVAS program, which also demonstrated a reduced risk of MACE compared with placebo [14]. More recently, the DECLARETIMI 58 study [15] showed that dapagliflozin reduced the risk of the combined endpoint of $\mathrm{CV}$ death or HHF, but showed non-inferiority for 3P-MACE. Results for the glucagon-like peptide 1 receptor agonist (GLP-1 RA) CVOTs have been mixed. LEADER [16], SUSTAIN-6 [17], Harmony Outcomes [18], and REWIND [19] demonstrated superiority for the composite MACE outcome vs. standard care. In contrast, other GLP-1 RA CVOTs demonstrated non-inferiority, including ELIXA [20], EXSCEL [21], and PIONEER 6 [22]. 
Table 1 Results of CVOTs for non-insulin glucose-lowering agents included in survey

\begin{tabular}{|c|c|c|c|c|}
\hline Study acronym & Agent studied & $\begin{array}{l}\text { MACE }^{a} \\
\text { HR ( } 95 \% \text { CI }) \\
P \text { value }\end{array}$ & $\begin{array}{l}\text { HHF } \\
\text { HR }(95 \% \mathrm{CI}) \\
P \text { value }\end{array}$ & $\begin{array}{l}\text { CV mortality } \\
\text { HR }(95 \% \text { CI }) \\
P \text { value }\end{array}$ \\
\hline \multicolumn{5}{|l|}{ DPP4 inhibitors } \\
\hline \multirow[t]{2}{*}{ SAVOR-TIMI 53 [6] } & Saxagliptin & $1.00(0.89-1.12)$ & $1.27(1.07-1.51)$ & $1.03(0.87-1.22)$ \\
\hline & & 0.99 & 0.007 & 0.72 \\
\hline \multirow[t]{2}{*}{ EXAMINE $[8,9]$} & Alogliptin & $0.96(\leq 1.16)$ & $1.07(0.79-1.46)$ & $0.79(0.60-1.04)$ \\
\hline & & 0.32 & 0.657 & 0.10 \\
\hline \multirow[t]{2}{*}{ TECOS [7] } & Sitagliptin & $0.98(0.89-1.08)^{\mathrm{c}}$ & $1.00(0.83-1.20)$ & $1.03(0.89-1.19)$ \\
\hline & & 0.65 & 0.98 & 0.71 \\
\hline \multirow[t]{2}{*}{ CARMELINA [10] } & Linagliptin & $1.02(0.89-1.17)$ & $0.90(0.74-1.08)$ & $0.96(0.81-1.14)$ \\
\hline & & 0.74 & 0.26 & 0.63 \\
\hline \multirow[t]{2}{*}{ CAROLINA [11] } & Linagliptin $^{\mathrm{d}}$ & $0.98(0.84-1.14)$ & $1.21(0.92-1.59)$ & $1.00(0.81-1.24)$ \\
\hline & & 0.76 & $0.18^{\mathrm{e}}$ & 0.99 \\
\hline \multicolumn{5}{|l|}{ SGLT2 inhibitors } \\
\hline \multirow[t]{2}{*}{ EMPA-REG OUTCOME $[12,13]$} & Empagliflozin & $0.86(0.74-0.99)$ & $0.65(0.50-0.85)$ & $0.62(0.49-0.77)$ \\
\hline & & 0.04 & 0.002 & $<0.001$ \\
\hline \multirow[t]{2}{*}{ CANVAS [14] } & Canagliflozin & $0.86(0.75-0.97)$ & $0.67(0.52-0.87)$ & $0.87(0.72-1.06)$ \\
\hline & & 0.02 & - & - \\
\hline \multirow[t]{2}{*}{ DECLARE-TIMI 58 [15] } & Dapagliflozin & $0.93(0.84-1.03)$ & $0.73(0.61-0.88)$ & $0.98(0.82-1.17)$ \\
\hline & & 0.17 & - & - \\
\hline \multicolumn{5}{|l|}{ GLP-1 RAs } \\
\hline \multirow[t]{2}{*}{ LEADER [16] } & Liraglutide & $0.87(0.78-0.97)$ & $0.87(0.73-1.05)$ & $0.78(0.66-0.93)$ \\
\hline & & 0.01 & 0.14 & 0.007 \\
\hline \multirow[t]{2}{*}{ ELIXA [20] } & Lixisenatide & $1.02(0.89-1.17)$ & $0.96(0.75-1.23)$ & $0.98(0.78-1.22)$ \\
\hline & & 0.81 & 0.75 & 0.85 \\
\hline \multirow[t]{2}{*}{ EXSCEL [21] } & Exenatide & $0.91(0.83-1.00)$ & $0.94(0.78-1.13)$ & $0.88(0.76-1.02)$ \\
\hline & & 0.06 & - & - \\
\hline \multirow[t]{2}{*}{ SUSTAIN-6 [17] } & Semaglutide & $0.74(0.58-0.95)$ & $1.11(0.77-1.61)$ & $0.98(0.65-1.48)$ \\
\hline & & 0.02 & 0.57 & 0.92 \\
\hline
\end{tabular}


Table 1 continued

\begin{tabular}{lllll}
\hline Study acronym & Agent studied & $\begin{array}{l}\text { MACE }^{\text {a }} \\
\text { HR (95\% CI) } \\
\boldsymbol{P} \text { value }\end{array}$ & $\begin{array}{l}\text { HHF } \\
\text { HR (95\% CI) } \\
\boldsymbol{P} \text { value }\end{array}$ & $\begin{array}{l}\text { CV mortality } \\
\text { HR (95\% CI) } \\
\boldsymbol{P} \text { value }\end{array}$ \\
\hline REWIND [19] & Dulaglutide & $0.88(0.79-0.99)$ & $0.93(0.77-1.12)^{\mathrm{e}}$ & $0.91(0.78-1.06)$ \\
& & 0.026 & 0.46 & 0.21 \\
\hline
\end{tabular}

Physicians were asked about their familiarity with these 13 CVOTs and VERTIS-CV; results for CARMELINA, CAROLINA, DECLARE-TIMI 58, REWIND, and VERTIS-CV were not available at the time the survey was conducted. Results for VERTIS-CV (ertugliflozin) were not available at the time of this writing

3P-MACE 3-point major adverse cardiovascular event, $4 P$-MACE 4-point major adverse cardiovascular event, $C I$ confidence interval, $C V$ cardiovascular, $C V O T$ cardiovascular outcomes trial, DPP4 dipeptidyl peptidase 4, GLP-1 RA glucagon-like peptide 1 receptor agonist, $H H F$ hospitalization for heart failure, $H R$ hazard ratio, $S G L T 2$ sodium-glucose co-transporter 2, SAVOR-TIMI 53 Saxagliptin Assessment of Vascular Outcomes Recorded in Patients with Diabetes Mellitus-Thrombolysis in Myocardial Infarction 53, TECOS Trial Evaluating Cardiovascular Outcomes with Sitagliptin, EXAMINE Examination of Cardiovascular Outcomes with Alogliptin Versus Standard of Care, CARMELINA Cardiovascular and Renal Microvascular Outcome Study with Linagliptin, CAROLINA Cardiovascular Outcome Study of Linagliptin vs Glimepiride in Type 2 Diabetes, EMPA-REG OUTCOME Empagliflozin Cardiovascular Outcome Event Trial in Type 2 Diabetes Mellitus Patients-Removal of Excess Glucose, CANVAS Canagliflozin Cardiovascular Assessment Study, DECLARETIMI 58 Dapagliflozin Effect on Cardiovascular Events-Thrombolysis in Myocardial Infarction 58, LEADER Liraglutide Effect and Action in Diabetes: Evaluation of Cardiovascular Outcome Results, SUSTAIN-6 Trial to Evaluate Cardiovascular and Other Long-term Outcomes with Semaglutide in Subjects with Type 2 Diabetes, REWIND Researching Cardiovascular Events with a Weekly Incretin in Diabetes, ELIXA Evaluation of Lixisenatide in Acute Coronary Syndrome, EXSCEL Exenatide Study of Cardiovascular Event Lowering, VERTIS-CV Evaluation of Ertugliflozin Efficacy and SafetyCardiovascular

a 3P-MACE (CV death, myocardial infarction, or stroke) unless otherwise specified

b $P$ value for superiority test; all CVOTs in this table demonstrated that they were non-inferior to placebo (for CAROLINA, non-inferior to glimepiride) as defined by an upper boundary of the $95 \% \mathrm{CI}$ for the $\mathrm{HR}<1.3$

c 4P-MACE (CV death, myocardial infarction, unstable angina, stroke)

${ }^{\mathrm{d}}$ Linagliptin vs. glimepiride

${ }^{\mathrm{e}}$ Hospital visit for heart failure or urgent visit

Results from these trials have led to changes in treatment guidelines and therapeutic indications for some glucose-lowering agents $[23,24]$. Little is known about primary care providers' knowledge of recent CVOTs and practice guidelines for the management of patients with T2DM. This survey was aimed at US IM and FM licensed physicians to examine their knowledge and beliefs about CVOTs for non-insulin glucose-lowering agents.

\section{METHODS}

The survey included $253 \mathrm{IM}$ and $250 \mathrm{FM}$ physicians from a database of 47,534 Medscape members who were invited to participate in an online survey via e-mail. To be eligible, physicians had to be a licensed MD (Doctor of Medicine) or DO (Doctor of Osteopathic Medicine) in the USA or its territories, board-eligible or board-certified (self-reported), see patients for at least $75 \%$ of the working week, and treat at least 10 patients with T2DM per week. Physicians were excluded from participation if they were from Vermont and Maine because of state laws regarding this type of research. The survey was fielded September 18-20, 2018; a quota of 500 responses was set. The questionnaire consisted of 23 questions. The first section assessed how familiar physicians were with 14 CVOTs, and their knowledge of the primary MACE 
A

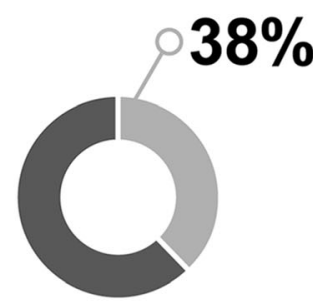

EMPA-REG OUTCOME (empagliflozin)

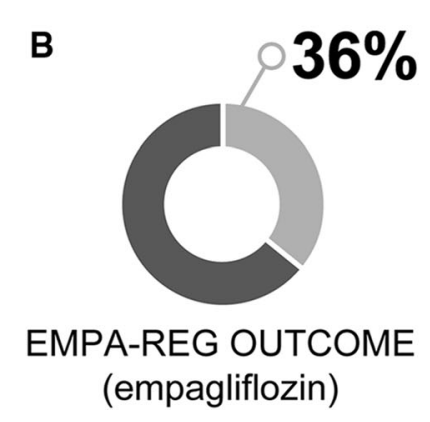

EMPA-REG OUTCOME (empagliflozin)

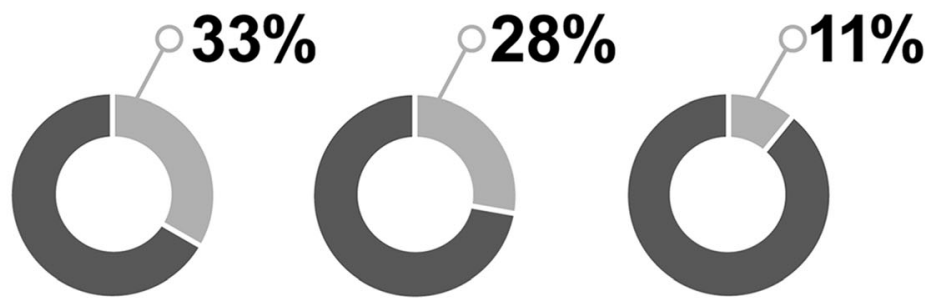

LEADER

CANVAS

(canagliflozin)

(liraglutide)

SUSTAIN-6

(semaglutide)

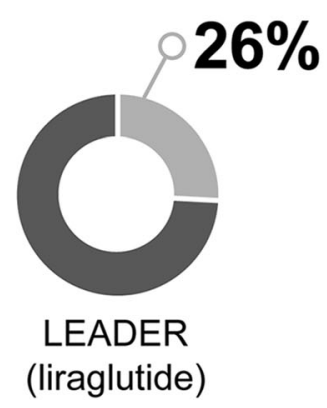

Fig. 1 Percentage of physicians who correctly responded that these CVOTs showed superiority for MACE (a) or CV death (b). CV cardiovascular, CVOT cardiovascular outcomes trial, MACE major adverse cardiovascular event

outcome, secondary outcomes, and safety signals. Physicians ranked how familiar they were with the 2018 American Diabetes Association (ADA) Standards of Medical Care in Diabetes treatment recommendations for individuals with atherosclerotic CV disease and T2DM [25]. The survey also asked physicians their opinions about management of their patients with T2DM and CV disease, and to identify the challenges they face when incorporating new recommendations from guidelines into their practice. Survey items were multiple choice and most used a 5-point Likert-type scale. Responses to each item were summarized and analyzed descriptively. A Z-test was used for statistical comparisons. Calculations were performed using Decipher software. The study protocol met the criteria for exemption by the Touro University of California Institutional Review Board (TUC IRB \#M-3118). Participants were informed about the intentions of the research and how their personal information and responses would be used and their confidentiality protected; informed consent was provided via a tick box at the beginning of the survey.

\section{RESULTS}

A total of 702 physicians responded, of whom 199 did not meet eligibility criteria. Data were collected from 503 physicians (IM, $n=253$; FM, $n=250$ ) who met screening criteria and completed the survey.

\section{Familiarity with CVOTs and Knowledge of MACE}

Familiarity with CVOTs was low. Respondents were most familiar with the three CVOTs that had positive $\mathrm{CV}$ outcomes reported at the time of the survey (LEADER, 39\%; EMPA-REG OUTCOME, 33\%; CANVAS, 30\%) as well as TECOS, $42 \% \quad(P<0.05$ vs. other CVOTs). Physicians were least familiar with EXAMINE, ELIXA, and VERTIS-CV ( $P<0.05$ vs. Other CVOTs). All of the CVOTs that had been reported at the time of the survey demonstrated at least non-inferiority 
(i.e., no difference in risk between the active agent and placebo) for the time to first occurrence of $\mathrm{CV}$ death, non-fatal myocardial infarction, or non-fatal stroke \pm hospitalization for unstable angina (MACE endpoint). At the time of the survey, four trials showed superiority (i.e., decreased risk vs. placebo) for the composite MACE endpoint; fewer than half of respondents identified those as EMPA-REG OUTCOME, LEADER, CANVAS, and SUSTAIN-6 (Fig. 1a). Similarly, $36 \%$ and $26 \%$ correctly chose EMPA-REG OUTCOME and LEADER, respectively, as showing superiority vs. placebo for CV mortality (Fig. 1b). Only $21 \%$ and $16 \%$ selected that EMPA-REG OUTCOME and CANVAS, respectively, showed superiority for HHF.

\section{Knowledge of Adverse Events in CVOTs}

Knowing the adverse events of the different classes of glucose-lowering agents is essential when choosing the appropriate treatment for patients with T2DM and CV disease. Acute pancreatitis is a labeled warning and precaution in the prescribing information for incretinbased therapies (DPP4 inhibitors and GLP-1 RAs). However, no significant differences in the incidence of acute pancreatitis between DPP4 inhibitor and placebo groups were observed in SAVOR-TIMI 53, TECOS, and EXAMINE [6-8]. Respondents were unaware of these findings; 20\% thought all three CVOTs showed a significant increase in the risk of acute pancreatitis. Similarly, no significant differences in the incidence of pancreatitis were observed between groups in the GLP-1 RA CVOTs [16, 17, 20, 21]. For this adverse event, $18 \%$ thought all of the listed GLP-1 RA CVOTs showed a significant increase in risk. Among the GLP-1 RA CVOTs, the rates of retinopathy complications were significantly higher in the SUSTAIN-6 trial (HR 1.76 ; $95 \%$ CI $1.11,2.78$ ) [17]. Only $8 \%$ of respondents selected that retinopathy occurred more frequently with semaglutide than placebo in SUSTAIN-6.

In the CANVAS program CVOT, an increased risk of lower limb amputations was observed [14]. This was not well recognized by respondents, with $36 \%$ selecting CANVAS alone for this finding. Empagliflozin use in EMPA-REG OUTCOME was not associated with an increased risk of lower limb amputations compared with placebo [26]. The majority of respondents correctly recognized this with only 8\% selecting EMPA-REG OUTCOME

\section{Beliefs and Challenges Regarding Treatment Priorities}

When provided with a list of seven treatment priorities ranked from most (1) to least (7) important, 33\% of respondents ranked using T2DM medications with $\mathrm{CV}$ benefits as least important, and 31\% ranked managing $\mathrm{CV}$ risk in general as most important $(P<0.05$ vs. all other categories). The main challenges primary care providers experienced using T2DM agents with $\mathrm{CV}$ benefits included insurance coverage (75\%), cost $(73 \%)$, and formulary changes (61\%).

\section{DISCUSSION}

Results for some CVOTs were not available at the time the survey was conducted in September 2018 (e.g., CAROLINA, CARMELINA, REWIND, DECLARE-TIMI 58). Nevertheless, this survey showed that in 2018 most IM and FM physicians were not familiar with the findings of recent CVOTs, despite the impact these trials have had on clinical practice guidelines for patients with T2DM at high CV risk. These findings are important as about one third of respondents made reducing $\mathrm{CV}$ risk their top priority but did not prioritize agents that have been shown to reduce risk.

Most physicians were unaware of the reduced risk of MACE events demonstrated for active treatment vs. placebo in EMPA-REG OUTCOME, LEADER, CANVAS, and SUSTAIN-6, and the reduced risk of HHF demonstrated in EMPA-REG OUTCOME and CANVAS. As these medications can impact the number one cause of death in people with diabetes, it is important to reconsider how results from CVOTs are disseminated to primary care providers.

Diabetes guidelines, such as the 2020 ADA Standards of Care [27] and the 2019 Consensus 
Statement from the American Association of Clinical Endocrinologists [28], explicitly recommend SGLT2 inhibitors and GLP-1 RAs with $\mathrm{CV}$ benefits for patients with these conditions. Nevertheless, a recent nationwide cohort study that retrospectively analyzed medical and pharmacy claims from 1,054,727 adults with pharmacologically treated diabetes revealed that patients with prior myocardial infarction or heart failure were less likely to receive SGLT2 inhibitors (OR 0.94 and 0.93, respectively, $P<0.001$ for both), despite evidence supporting their preferential use in these patients [29].

With respect to adverse events, physicians tended to overestimate the risk of pancreatitis with DPP4 inhibitors and GLP-1 RAs. In contrast, there was under-recognition of the increased risk of retinopathy complications associated with semaglutide in SUSTAIN-6, and the increased rates of lower limb amputations associated with canagliflozin in the CANVAS program.

While it takes time for new scientific findings to be implemented in daily practice, physicians who do not use guideline-recommended approaches to $\mathrm{CV}$ risk reduction in patients with T2DM at increased $\mathrm{CV}$ risk are likely to miss the opportunity to achieve reductions in CV-related morbidity and mortality for their patients.

There are several limitations in this US study, such as the relatively small sample size that may not be representative of all IM and FM physicians. Because participants were self-selected Medscape users who responded to an email invitation, it is not possible to exclude the potential for bias by over-representation of individuals with similar characteristics. However, the findings of this survey can provide information for hypothesis generation and stimulus for further research. The survey also was conducted at a single point in time in 2018. As additional studies have been published that confirm the CV benefits of SGLT2 inhibitors and GLP-1 RAs, IM, and FM physicians likely have increased awareness of CVOTs compared with when the survey was originally conducted.

In conclusion, the findings from this survey suggest knowledge gaps among IM and FM physicians about CVOTs, which have implications for the treatment of patients with T2DM and CV disease. There is a need for enhanced training and dissemination of clinical practice guidelines to ensure that patients with T2DM and $\mathrm{CV}$ risk can benefit from evidence-based therapies.

\section{ACKNOWLEDGEMENTS}

We thank the survey respondents for their participation in the study.

Funding. This article was supported by Boehringer Ingelheim Pharmaceuticals, Inc., Ridgefield, CT, USA. The sponsor has also funded the journal's Rapid Service and Open Access fees.

Medical Writing Assistance. Writing support was provided by Jennifer Garrett, MBBS, Linda Merkel PhD, and Marissa Buttaro, MPH, of Elevate Scientific Solutions, which was contracted and compensated by Boehringer Ingelheim Pharmaceuticals, Inc. (BIPI) for this service.

Authorship. The authors meet criteria for authorship as recommended by the International Committee of Medical Journal Editors (ICMJE), and were fully responsible for all content and editorial decisions, were involved at all stages of manuscript development, and approved the final version that reflects the authors' interpretations and conclusions. The authors received no direct compensation related to the development of the manuscript.

Prior Presentations. Data have been previously presented at the Society of General Internal Medicine (SGIM) 2019 Annual Meeting, May 8-11, 2019, Washington, DC, USA; and an abstract (\#2226) was published in Diabetes 2019 Jun; 68(Supplement 1).

Disclosures. Jay H. Shubrook has received research support from Lilly Diabetes and Sanofi, and served as a consultant to Lilly, Novo Nordisk, Intarcia, Bayer, and Sanofi. Jonathan Pak is an employee of BIPI. George Dailey has attended speakers' bureaus for AstraZeneca and 
Sanofi; received research support from Eli Lilly, Janssen, Mylan, Novo Nordisk, and Sanofi; and received consulting fees from Boehringer Ingelheim, Novo Nordisk, and Sanofi.

Compliance with Ethics Guidelines. The study protocol met the criteria for exemption by the Touro University of California Institutional Review Board (TUC IRB \#M-3118). Participants were informed about the intentions of the research and how their personal information and responses would be used and their confidentiality protected; informed consent was provided via a tick box at the beginning of the survey.

Data Availability. The datasets generated during and/or analyzed during the current study, including the original survey, are available from the corresponding author on reasonable request.

Open Access. This article is licensed under a Creative Commons Attribution-NonCommercial 4.0 International License, which permits any non-commercial use, sharing, adaptation, distribution and reproduction in any medium or format, as long as you give appropriate credit to the original author(s) and the source, provide a link to the Creative Commons licence, and indicate if changes were made. The images or other third party material in this article are included in the article's Creative Commons licence, unless indicated otherwise in a credit line to the material. If material is not included in the article's Creative Commons licence and your intended use is not permitted by statutory regulation or exceeds the permitted use, you will need to obtain permission directly from the copyright holder. To view a copy of this licence, visit http://creativecommons.org/licenses/by$\mathrm{nc} / 4.0 /$.

\section{REFERENCES}

1. American Diabetes Association. 10. Cardiovascular disease and risk management: standards of medical care in diabetes. Diabetes Care. 2020;43:S111-134.
2. Huxley R, Barzi F, Woodward M. Excess risk of fatal coronary heart disease associated with diabetes in men and women: meta-analysis of 37 prospective cohort studies. BMJ. 2006;332:73-8.

3. Marx N, McGuire DK, Perkovic V, et al. Composite primary end points in cardiovascular outcomes trials involving type 2 diabetes patients: should unstable angina be included in the primary end point? Diabetes Care. 2017;40:1144-51.

4. Cefalu WT, Kaul S, Gerstein HC, et al. Cardiovascular outcomes trials in type 2 diabetes: where do we go from here? Reflections from a Diabetes Care editors' expert forum. Diabetes Care. 2018;41: 14-31.

5. Home P. Cardiovascular outcome trials of glucoselowering medications: an update. Diabetologia. 2019;62:357-69.

6. Scirica BM, Bhatt DL, Braunwald E, et al. Saxagliptin and cardiovascular outcomes in patients with type 2 diabetes mellitus. N Engl J Med. 2013;369: 1317-26.

7. Green JB, Bethel MA, Armstrong PW, et al. Effect of sitagliptin on cardiovascular outcomes in type 2 diabetes. N Engl J Med. 2015;373:232-42.

8. White WB, Cannon CP, Heller SR, et al. Alogliptin after acute coronary syndrome in patients with type 2 diabetes. N Engl J Med. 2013;369:1327-35.

9. Zannad F, Cannon CP, Cushman WC, et al. Heart failure and mortality outcomes in patients with type 2 diabetes taking alogliptin versus placebo in EXAMINE: a multicentre, randomised, doubleblind trial. Lancet. 2015;385:2067-76.

10. Rosenstock J, Perkovic V, Johansen OE, et al. Effect of linagliptin vs placebo on major cardiovascular events in adults with type 2 diabetes and high cardiovascular and renal risk: the CARMELINA randomized clinical trial. JAMA. 2019;321:69-79.

11. Rosenstock J, Kahn SE, Johansen OE, et al. Effect of linagliptin vs glimepiride on major adverse cardiovascular outcomes in patients with type 2 diabetes: the CAROLINA randomized clinical trial. JAMA. 2019;322:1155-66.

12. Fitchett D, Inzucchi SE, Lachin JM, et al. Cardiovascular mortality reduction with empagliflozin in patients with type 2 diabetes and cardiovascular disease. J Am Coll Cardiol. 2018;71:364-7.

13. Zinman B, Wanner C, Lachin JM, et al. Empagliflozin, cardiovascular outcomes, and mortality in type 2 diabetes. N Engl J Med. 2015;373:2117-28. 
14. Neal B, Perkovic V, Mahaffey KW, et al. Canagliflozin and cardiovascular and renal events in type 2 diabetes. N Engl J Med. 2017;377:644-57.

15. Wiviott SD, Raz I, Bonaca MP, et al. Dapagliflozin and cardiovascular outcomes in type 2 diabetes. N Engl J Med. 2019;380:347-57.

16. Marso SP, Daniels GH, Brown-Frandsen K, et al. Liraglutide and cardiovascular outcomes in type 2 diabetes. N Engl J Med. 2016;375:311-22.

17. Marso SP, Bain SC, Consoli A, et al. Semaglutide and cardiovascular outcomes in patients with type 2 diabetes. N Engl J Med. 2016;375:1834-44.

18. Hernandez AF, Green JB, Janmohamed S, et al. Albiglutide and cardiovascular outcomes in patients with type 2 diabetes and cardiovascular disease (Harmony Outcomes): a double-blind, randomised placebo-controlled trial. Lancet. 2018;392:1519-29.

19. Gerstein HC, Colhoun HM, Dagenais GR, et al. Dulaglutide and cardiovascular outcomes in type 2 diabetes (REWIND): a double-blind, randomised placebo-controlled trial. Lancet. 2019;394:121-30.

20. Pfeffer MA, Claggett B, Diaz R, et al. Lixisenatide in patients with type 2 diabetes and acute coronary syndrome. N Engl J Med. 2015;373:2247-57.

21. Holman RR, Bethel MA, Mentz RJ, et al. Effects of once-weekly exenatide on cardiovascular outcomes in type 2 diabetes. N Engl J Med. 2017;377:1228-39.

22. Husain M, Birkenfeld AL, Donsmark M, et al. Oral semaglutide and cardiovascular outcomes in patients with type 2 diabetes. $\mathrm{N}$ Engl J Med. 2019;381:841-51.
23. American Diabetes Association. 10. CV disease and risk management: standards of medical care in diabetes-2019. Diabetes Care. 2019;42(Suppl 1): S103-23.

24. Cosentino F, Grant PJ, Aboyans V, et al. 2019 ESC guidelines on diabetes, pre-diabetes, and cardiovascular diseases developed in collaboration with the EASD. Eur Heart J. 2020;41:255-323.

25. American Diabetes Association. 9. Pharmacologic approaches to glycemic treatment: standards of medical care in diabetes. Diabetes Care. 2018;41: S86-104.

26. Inzucchi SE, Iliev H, Pfarr E, Zinman B. Empagliflozin and assessment of lower-limb amputations in the EMPA-REG OUTCOME trial. Diabetes Care. 2018;41:e4-5.

27. American Diabetes Association. Standards of medical care in diabetes 2020. Diabetes Care. 2020;43: S1-212.

28. Garber AJ, Handelsman Y, Grunberger G, et al. Consensus statement by the American Association of Clinical Endocrinologists and American College of Endocrinology on the comprehensive type 2 diabetes management algorithm-2020 executive summary. Endocr Pract. 2020;26:107-39.

29. McCoy RG, Dykhoff HJ, Sangaralingham L, et al. Adoption of new glucose-lowering medications in the US-the case of SGLT2 inhibitors: nationwide cohort study. Diabetes Technol Ther. 2019;21: 702-12. 